\title{
A LOCAL MEAN-VALUE THEOREM FOR ANALYTIC FUNCTIONS WITH SMOOTH BOUNDARY VALUES
}

\author{
by W. P. NOVINGER
}

(Received 11 September, 1972)

Let $f$ be an analytic function on a connected open set $\Omega$ in the complex plane. Then, for given $a, b \in \Omega$, the equation

$$
(b-a) f^{\prime}(z)=f(b)-f(a)
$$

need not have a solution $z \in \Omega$. As a matter of fact, this would happen with each locally oneto-one analytic function which is not one-to-one on $\Omega$. But if we fix $a \in \Omega$, then, for all $b$ sufficiently close to $a,(1)$ is solvable for $z$. This is an easy consequence of the Open Mapping Theorem applied to $f^{\prime}$. For, assuming that $f^{\prime}$ is non-constant (otherwise, (1) holds for all $a, b, z \in \Omega)$, the Open Mapping Theorem tells us that $f^{\prime}(\Omega)$, the image under $f^{\prime}$ of $\Omega$, is an open neighbourhood of $f^{\prime}(a)$; so it is a direct consequence of the definition of $f^{\prime}(a)$ that there exists $\delta>0$ such that $0<|b-a|<\delta$ implies $(f(b)-f(a)) /(b-a) \in f^{\prime}(\Omega)$. A stronger statement has been obtained by J. M. Robertson [1, p. 329], who has shown that

$$
(f(b)-f(a)) /(b-a) \in f^{\prime}(\{z:|z-a|<|b-a|\}),
$$

and, if $f^{\prime \prime}(a) \neq 0$, then

$$
(f(b)-f(a)) /(b-a) \in f^{\prime}\left(\left\{z:\left|z-\frac{1}{2}(a+b)\right|<\frac{1}{2}|a-b|\right\}\right) .
$$

Recently, Åke Samuelsson [3] has succeeded in removing the restriction $f^{\prime \prime}(a) \neq 0$. He proves that, if $n$ is the smallest positive integer such that $f^{(n+1)}(a) \neq 0$ (so that $f^{\prime}-f^{\prime}(a)$ has a zero of order $n$ at $a$ ), then

$$
\frac{f(b)-f(a)}{b-a} \in f^{\prime}\left(\left\{z:\left|z-\frac{1}{2}(a+b)\right|<\frac{1}{2}|a-b| \text { and }\left|\arg \frac{z-a}{b-a}\right|<\frac{\pi}{n}\right\}\right) \text {. }
$$

A close examination of Samuelsson's argument shows that $\pi / n$ can be improved to $\pi / 2 n$ in (2). The purpose of the present article is to investigate the validity of the sharpened version of (2) when $a$ is a boundary point of $\Omega, f$ satisfies a continuity or smoothness condition at $a$, and then $b \in \Omega$ is sufficiently close to $a$.

Definimion. For distinct complex numbers $a$ and $b$, and positive integers $n, K_{n}(a ; b)$ will denote the region consisting of

$$
\left\{z:\left|z-\frac{1}{2}(a+b)\right|<\frac{1}{2}|a-b| \text { and }\left|\arg \frac{z-a}{b-a}\right|<\frac{\pi}{2 n}\right\} \text {. }
$$

THEOREM. Suppose that $f$ is analytic on a region $\Omega, a$ is a boundary point of $\Omega$, and, for some positive integer $n, f^{(n+1)}$ has a continuous extension to $\Omega \cup\{a\}$ with $f^{(n+1)}(a) \neq 0$. If $n$ is the smallest such integer, then there exists $\delta>0$ such that, if $b \in \Omega,|b-a|<\delta$ and $\bar{K}_{n}(a ; b) \subset$ $\Omega \cup\{a\}$, then $(f(b)-f(a)) /(b-a) \in f^{\prime}\left(K_{n}(a ; b)\right)$. 
Notes. (a) The hypothesis implies that each of $f, f^{\prime}, \ldots, f^{(n)}$ has extensions to $\Omega \cup\{a\}$ such that, for $k=0,1, \ldots, n,\left(f^{(k)}(z)-f^{(k)}(a)\right) /(z-a) \rightarrow f^{(k+1)}(a)$ as $z \rightarrow a, z \in \Omega$. (b) Samuelsson's result is the special case where $\Omega \cup\{a\}$ is a neighbourhood of $a$ in the complex plane.

Proof of Theorem. Choose $\delta>0$ such that $z \in \Omega$ and $|z-a|<\delta$ imply

$$
(n+2)\left|f^{(n+1)}(z)-f^{(n+1)}(a)\right|<\left|f^{(n+1)}(a)\right| \text {. }
$$

Assume that $b \in \Omega,|b-a|<\delta$, and $\bar{K}_{n}(a ; b) \subset \Omega \cup\{a\}$. Then, for $z \in \bar{K}_{n}(a ; b)$,

$$
f(z)=f(a)+f^{\prime}(a)(z-a)+\frac{1}{n !} \int_{a}^{z} f^{(n+1)}(\tau)(z-\tau)^{n} d \tau,
$$

where the integral is taken along the straight line segment from $a$ to $z$. Thus we can write

$$
\begin{aligned}
& f^{\prime}(z)-\frac{f(b)-f(a)}{b-a}=\frac{1}{(n-1) !} \int_{a}^{z} f^{(n+1)}(\tau)(z-\tau)^{n-1} d \tau-\frac{(b-a)^{-1}}{n !} \int_{a}^{b} f^{(n+1)}(\tau)(b-\tau)^{n} d \tau \\
& =\frac{f^{(n+1)}(a)}{n !}(z-a)^{n}+\frac{1}{(n-1) !} \int_{a}^{z}\left[f^{(n+1)}(\tau)-f^{(n+1)}(a)\right](z-\tau)^{n-1} d \tau \\
& -\frac{f^{(n+1)}(a)}{(n+1) !}(b-a)^{n}-\frac{(b-a)^{-1}}{n !} \int_{a}^{b}\left[f^{(n+1)}(\tau)-f^{(n+1)}(a)\right](b-\tau)^{n} d \tau \\
& =F(z)+G(z), \\
& F(z)=\frac{f^{(n+1)}(a)}{(n+1) !}\left[(n+1)(z-a)^{n}-(b-a)^{n}\right] \\
& G(z)=\frac{1}{(n-1) !} \int_{a}^{z}\left[f^{(n+1)}(\tau)-f^{(n+1)}(a)\right](z-\tau)^{n-1} d \tau \\
& -\frac{(b-a)^{-1}}{n !} \int_{a}^{b}\left[f^{(n+1)}(\tau)-f^{(n+1)}(a)\right](b-\tau)^{n} d \tau .
\end{aligned}
$$

Plainly, $F$ and $G$ are continuous on $\bar{K}_{n}(a ; b)$ and analytic in $K_{n}(a ; b)$, and $F$ has exactly one zero in $K_{n}(a ; b)$. Thus Rouché's Theorem implies our result if we show that $|F(z)|>|G(z)|$ for $z$ on the boundary of $K_{n}(a ; b)$; proof of this follows. Now, if $z-a=\frac{1}{2}(b-a)+\frac{1}{2}(b-a) \exp (2 i \theta)$, where $|\theta| \leqq \pi / 2 n$, then

$$
\begin{aligned}
\left|(n+1)(z-a)^{n}-(b-a)^{n}\right| & =|b-a|^{n}\left|(n+1) \cos ^{n} \theta-\exp (i n \theta)\right| \\
& =|b-a|^{n}\left|(n+1) \cos ^{n} \theta-2 \cos n \theta+\exp (-i n \theta)\right| .
\end{aligned}
$$

Since $(n+1) \cos ^{n} \theta-2 \cos n \theta \geqq 0$ (this follows from the more general fact that $\cos ^{n} \theta \geqq \cos n \theta$ for $(n-1)|\theta| \leqq \pi)$, it is geometrically obvious that $\left|(n+1) \cos ^{n} \theta-2 \cos n \theta+\exp (-i n \theta)\right| \geqq 1$, so that $\left|(n+1)(z-a)^{n}-(b-a)^{n}\right| \geqq|b-a|^{n}$. If $z-a=t(b-a) \exp ( \pm(\pi i / 2 n))$ and $t \geqq 0$, then $\left|(n+1)(z-a)^{n}-(b-a)^{n}\right|=|b-a|^{n}\left|(n+1) t^{n} i-1\right| \geqq|b-a|^{n}$. It follows from the inequalities of the preceding two sentences and (4) that 


$$
|F(z)| \geqq\left|\frac{f^{(n+1)}(a)}{(n+1) !}\right||b-a|^{n}, \quad z \in \partial K_{n}(a ; b) .
$$

On the other hand, for $z \in \bar{K}_{n}(a ; b)$, the representation (5) and inequality (3) give

$$
|G(z)|<\frac{1}{n !} \cdot \frac{\mid f^{(n+1)}(a)}{n+2}|z-a|^{n}+\frac{1}{(n+1) !} \frac{\left|f^{(n+1)}(a)\right|}{n+2}|b-a|^{n} \leqq \frac{\left|f^{(n+1)}(a)\right|}{(n+1) !}|b-a|^{n} \text {. }
$$

Thus $F$ and $F+G$ have the same number of zeros in $K_{n}(a ; b)$. This completes the proof.

The example which follows shows that there is no possibility of establishing a result along the lines of that just proven if one merely assumes continuity at boundary points. In fact, there is a function $f$ which is continuous on the closure $\bar{U}$ of the unit disc $U$, is analytic in $U$, and is such that $f\left(a_{k}\right)=0$ for a sequence $\left\{a_{k}\right\}$ converging to $a \in \partial U$, while $f^{\prime}$ has no zeros in $U$. Such a function can be obtained as follows. For $z \neq 0$, define

$$
g(z)=z \sin (1 / z) \exp (i / z) \text {. }
$$

Then $g$ is analytic on its domain $\mathbb{C}\{0\}$, and it is readily verified that its derivative $g^{\prime}$ has no real zeros. It follows from this and the continuity of $g$ and $g^{\prime}$ that we can associate with each real number $x$, with $0<x<1$, a positive number $\delta_{x} \leqq x$ with the property that $|g(z)-g(x)|<x$ and $g^{\prime}(z) \neq 0$, for all $z$ satisfying $|z-x|<\delta_{x}$. Let $V=\bigcup\left\{z:|z-x|<\delta_{x}, 0<x<1\right\}$. If we define $g(0)=0$, then the restriction of $g$ to $\bar{V}$ is continuous. Further, it is routine to verify that $V$ is a bounded, simply connected region and that each boundary point is simple in the sense of [2, Definition 14.16, p. 279]. (Equivalently, $\partial V$ is a simple closed curve.) Thus, by a well-known theorem of Carathéodory [2, Theorem 14.19, p. 281], there is a homeomorphism $\phi$ of $\bar{U}$ onto $\bar{V}$ which is analytic on $U$. Then $f=g \circ \phi$ is continuous on $\bar{U}$ and analytic in $U$. If we set $a_{k}=\phi^{-1}(1 / k \pi)(k=1,2, \ldots)$, then $\left\{a_{k}\right\}$ converges to a point $a \in \partial U$, but $f^{\prime}=\left(g^{\prime} \circ \phi\right) \phi^{\prime}$ has no zeros in $U$. Hence some smoothness condition at $a$ is necessary. It remains an open question, however, whether or not the condition $f^{(n+1)}(a) \neq 0$ can be dropped from the hypothesis of the theorem (with, of course, an appropriate modification of the statement of the conclusion). An illustration of the situation which we have in mind is furnished by taking $\Omega=\{z:-\pi<\arg z<\pi\}$ and defining $f$ on $\Omega$ by $f(z)=\exp \left(-z^{-1 / 3}\right)$, where $1^{-1 / 3}=1$. This function has the property that it and all its derivatives have continuous extensions to $\Omega \cup\{0\}$, but $f^{(n+1)}(0)=0$ for each positive integer $n$. So our theorem gives no information regarding the existence or location of points $z$ such that $(b-a) f^{\prime}(z)=f(b)-f(a)$ when $a=0$ and $b \in \Omega$ is given. Of course, given $b$ real, $b f^{\prime}(z)=f(b)$ for some $z$ in the open interval $(0, b)$ by the classical mean value theorem.

\section{REFERENCES}

1. J. M. Robertson, A local mean value theorem for the complex plane, Proc. Edinburgh Math. Soc. (2) $16(1968 / 69), 329-331$.

2. Walter Rudin, Real and Complex Analysis (New York, 1966).

3. Ake Samuelsson, A local mean value theorem for analytic functions, Amer. Math. Monthly 30 (1973), 45.

\section{FloRida STATE University}

Tallahassee, Florida 32306 